\title{
Radar as an Emerging and Growing Technology for Industrial Applications: A Short Overview
}

Philipp Hügler, Martin Geiger, and Christian Waldschmidt

Institute of Microwave Engineering, Ulm University, 89081 UIm, Germany

paul.huegler@uni-ulm.de, martin-2.geiger@uni-ulm.de, christian.waldschmidt@uni-ulm.de

\begin{abstract}
:
After a brief introduction of classical radar applications, a short overview of state-of-the-art radar technology is presented. For basic radar understanding, the chirp-sequence frequency modulation scheme and its advantages are shortly described. As an outlook, four future application areas for modern radar sensors are shown. A $160 \mathrm{GHz}$ flexible sniffing probe is proposed for security and industrial applications. For human gesture recognition and unmanned aerial vehicle altimeter a $77 \mathrm{GHz}$ radar is presented. The ground penetrating capability of radar is shown, using a low frequency FMCW radar for buried landmine detection.
\end{abstract}

Key words: Chirp-sequence basic principle, $160 \mathrm{GHz}$ radar sniffer probe, gesture recognition, radar altimeter, ground penetrating radar

\section{Introduction}

Radar systems became popular through the use in military applications [1,2], civil air surveillance, and maritime surveillance [3]. Other well-known radar applications are weather radars $[4,5]$ or earth observation satellites [6]. In all these applications frequencies below $10 \mathrm{GHz}$ are used to determine ranges larger than $100 \mathrm{~km}$, velocities, and for imaging. The radar systems have bulky dimensions and a complex signal processing unit. Consequently, the systems are expensive and not attractive for industrial sensing or for private consumers.

In contrast to this, the robustness of radar in measuring distance, velocities, and angles compared to optical or ultrasound system is a preferable feature. With the possibility of integrating complete radar systems on chip (SoC) in silicon-germanium (SiGe) $[7,8]$, cheap production is feasible and radar systems become popular for industrial applications as well. Compactness is even more increased by integrating antennas in package resulting in fullyintegrated transceivers on a single chip, also eliminating the need of high frequency transitions for signals from chip to $\operatorname{PCB}[9,10]$.

The presence of radar in everyday life application is constantly increasing by now. Simple continuous wave (CW) sensors working in the $24 \mathrm{GHz}$ range, capable of detecting movement and measuring velocity, are for example used in automatic door openers [11]. More complex systems using frequencymodulated continuous wave (FMCW) sensors in the $77 \mathrm{GHz}$ range, able to measure distance, velocity, and increasingly also angular information, are used in the automotive industry. Many advanced driver assistance systems, like adaptive cruise control, collision avoidance, and blind spot monitoring mainly rely on radar data [12].

For industrial applications radar can be used for a large variety of applications. Because of its already mentioned robustness in harsh environments, it is used for low power level measurements in industrial tanks [13]. Compared to other filling level measurement techniques (e.g. ultrasound and optical) for applications with liquids radar can be used for nearly every viscosity of the measured medium and temperature range [14]. Furthermore, radar is used in mining where bulk material is won with bucket wheel reclaimers. Another application is the surveillance of conveyor belts in agriculture or the chemical industry to measure the load volume, the velocity, and misalignment. For the automation of loading large containers on ships, a radar is installed to avoid collisions and control the evenly charging. In all these applications, dust and weather conditions would be a limiting factor for other sensors. There are possibilities to gain high range detection in the $\mu \mathrm{m}$ range by combining frequency and phase evaluation with small bandwidths [15]. This can for example be used in placement and vibration measurements [16]. Other fields of applications are through-thewall inspection and ground penetrating radar for the detection of buried objects and bodies as well as nondestructive testing [17]. There are already commercial products like the D-tect 150 by Bosch, able to differentiate between several 


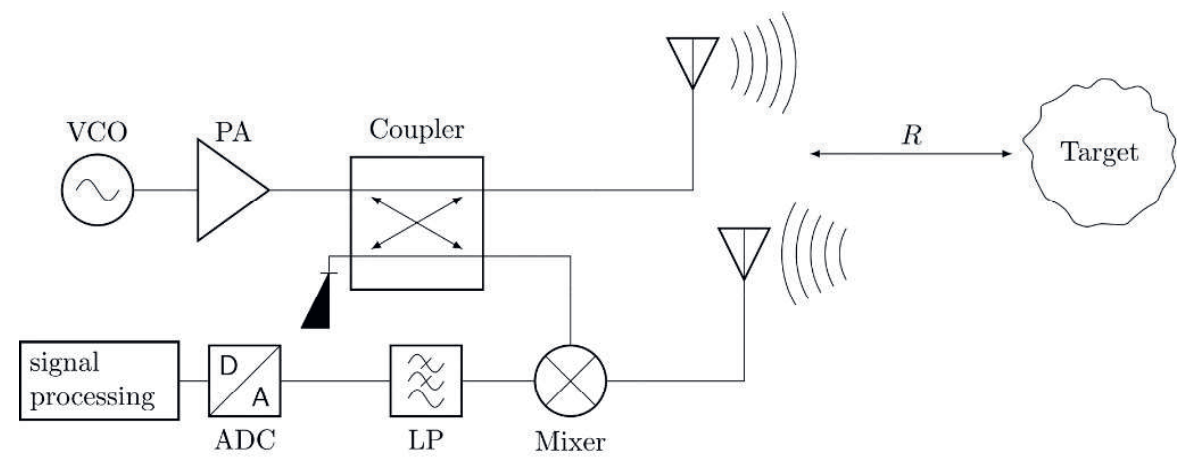

Fig. 1: Bi-static FMCW radar block diagram.

materials and measuring the size, position and depth of the corresponding object in a wall. In addition, it is also possible to get 3D images of objects in or behind a wall [18].

In this paper, a brief overview about the basic FMCW radar principles is given. After a short discussion of radar advantages several new radar applications are presented. One application is a radar system at $160 \mathrm{GHz}$ with a flexible measuring probe. Second gesture recognition with a radar system is presented. Another subject are radar systems mounted on unmanned aerial vehicles (UAV). On the one hand a $77 \mathrm{GHz}$ radar based altimeter and on the other hand a low-frequency ground penetrating radar for buried object recognition are presented.

\section{Basic Radar Principle}

To get familiar with the working principle of a FMCW radar, a basic block diagram of a bi-static radar is depicted in Fig. 1. The transmitted signal is generated by a voltage-controlled oscillator (VCO), with the tuning voltage being controlled by a phase-locked loop (PLL). The resulting RFsignal is amplified by a power amplifier (PA) and passed through a coupler, which divides the signal. One is radiated by the transmitting antenna, the other is passed to the receiving mixer as the local oscillator (LO) signal. At distance $R$ the transmitted signal is reflected by

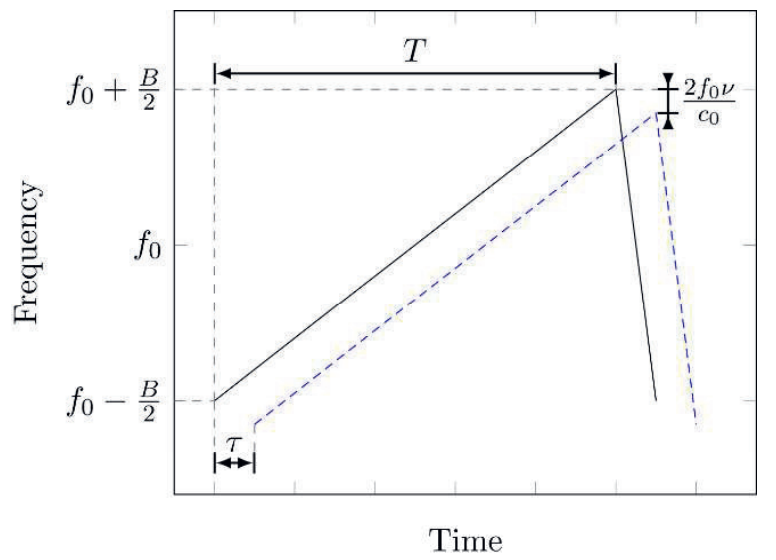

Fig. 2: Chirp sequence frequency modulation. a target with radar cross-section (RCS) $\sigma$ and received by the receiving antenna. Afterwards it is down-converted with the LO-signal, and after an anti-aliasing lowpass filter (LP) fed into an analog-to-digital converter (ADC). Assuming a point target, the received power $P_{\mathrm{RX}}$ at the feedpoint of the receiving antenna can be calculated with the so called radar equation:

$$
P_{\mathrm{RX}}=\frac{P_{\mathrm{TX}} G_{\mathrm{TX}} G_{\mathrm{RX}} \lambda^{2} \sigma}{(4 \pi)^{3} R^{4}}
$$

with $P_{\mathrm{TX}}$ the transmitted power, $G_{\mathrm{TX} / \mathrm{RX}}$ the gain of the transmitting/receiving antenna, and $\lambda$ the free-space wavelength.

For further investigations, chirp-sequence (CS) will be used as the frequency modulation since it is one of the most common modulation formats nowadays. With CS it is possible to measure distance and velocity of multiple targets (as well as the direction of arrival (DoA)) at the same time. Frequency is modulated as a linear ramp with bandwidth $B$ around the center frequency $f_{0}$ with duration $T$. Fig. 2 shows such a ramp (solid black curve). This ramp is repeated $L$-times with ramp repetition interval $T_{\mathrm{RRI}}$. Regarding the echo of the transmitted signal (blue dashed curve Fig. 2 ) reflected by a target with distance $R$ and velocity $v$, the ramp is shifted in time by the delay $\tau=2(R+v \cdot t) / c_{0}$ and in frequency by the Doppler frequency. In order to extract the target distance and velocity, the phase of the downconverted signal

$$
\Delta \phi(t)=\phi(t)-\phi(t-\tau)
$$

is analyzed as in [20]:

$$
\Delta \phi(t)=2 \pi(f_{0} \tau-\frac{B}{T} t \tau+\underbrace{\frac{B}{2 T} \tau^{2}}_{\approx 0 \text { for } \tau \ll T})
$$

The expected intermediate frequency (IF) $f_{\mathrm{IF}}$ is found by neglecting the $\tau^{2}$-term in (3) and inserting $\tau$ 


$$
f_{\mathrm{IF}}=\frac{1}{2 \pi} \frac{\mathrm{d} \Delta \phi(t)}{\mathrm{d} t}=\frac{2 f_{0} v}{c_{0}}+\frac{2 B R}{T c_{0}} .
$$

Based on the IF-signal, the range, as well as the velocity can be determined. In the first step, a fast-Fourier transformation (FFT) is performed over each ramp to determine the target distance (signal range gate). In the next step, a second FFT over all consecutive send $L$ ramps for each range bin is applied to calculate the target velocity. This procedure and the resulting rangeDoppler-matrix is shown in Fig. 3. The maximum Doppler frequency $f_{\mathrm{D} \text {, max }}$ is limited by $T_{\mathrm{RRI}}$ and the velocity resolution $\Delta v$ by the observation time:

$$
f_{\mathrm{D}, \max }<\frac{1}{2 T_{\mathrm{RRI}}}, \quad \Delta v=\frac{c_{0}}{2 f_{0} T_{\mathrm{RRI}} L} .
$$

The range resolution $\Delta R$ is inversely proportional to the measurement time available in one ramp, for $\tau \ll T, t_{\text {meas }} \approx T$, it can be derived that [21]:

$$
\Delta \mathrm{R} \geq \frac{c_{0} T}{2 B} \cdot \frac{1}{t_{\text {meas }}} \approx \frac{c_{0}}{2 B} .
$$

For DoA measurements, multiple receive channels are needed. In the easiest case, a uniform linear antenna array (ULA) is used, with an antenna spacing of half the free-space wavelength to avoid grating-lobes. Assuming a target in the far field, a plane wave is present at the antenna array. This leads to a phase difference between the consecutive channels. This phase difference is used to calculate the direction of arrival. There are many algorithms with different approaches, for example Bartlett beamformer, Maximum Likelihood (ML), and multiple signal classification (MUSIC) which will not be further investigated in this paper. More information can be found in [22, 23, 24].

\section{Radar Applications}

In the following sections a brief overview of new radar applications is given. To show the variety and advantages of radar, three different radar

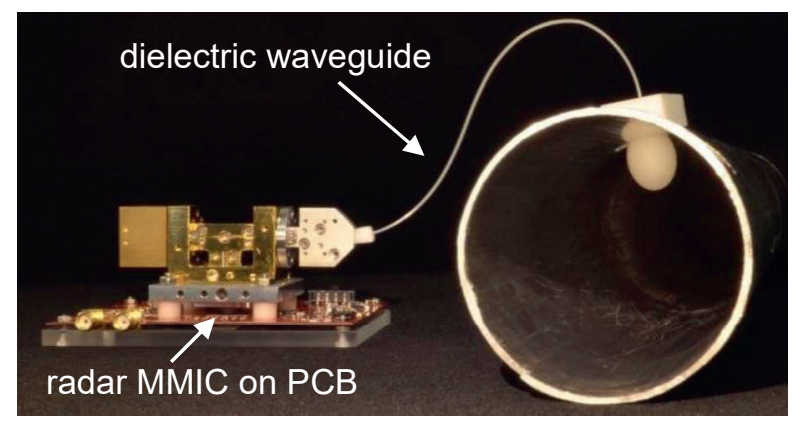

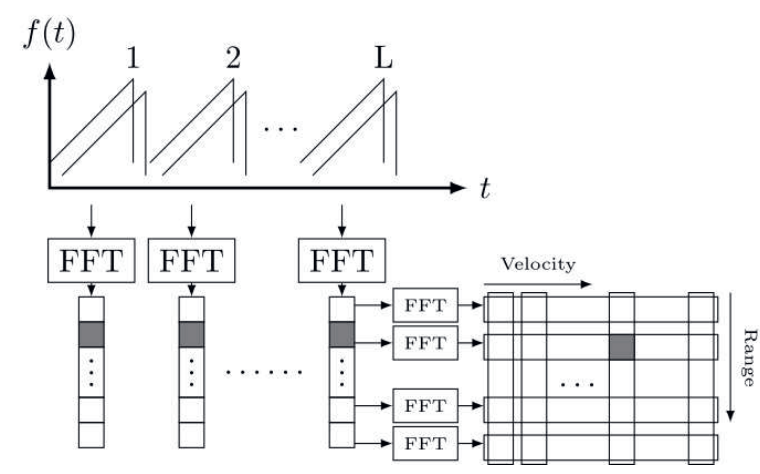

Fig. 3: Range-Doppler-matrix cf. [19].

systems at three different frequency bands are presented.

\section{GHz Sniffer Probe}

For frequencies above $100 \mathrm{GHz}$, radar systems are usually integrated systems on Microwave Monolithic Integrated Circuit (MMIC) with onchip antennas. Due to the limited gain of these antennas, MMICs are mainly used in combination with lenses above the chip. Such systems are large compared to the chip size and can only detect targets in the lens' beamwidth.

A system with a flexible sniffer probe as known from oscilloscopes offers new areas of application for radar. The known radar measurement scenarios as level measurement in industrial tanks can be extended to fill level and flow rate measurements in small pipes. Furthermore, the radar can be used in surroundings where due to space limitations or obstacles between sensor chip and target only an antenna, the sniffer, can be placed. The rest of the system is outside of these surroundings where enough space is available. Here security applications with endoscopes can be extended with range and velocity measurements. Another advantage of a flexible sniffer probe is the spatial separation of radar MMIC and antenna. For distance measurements at robot arms in environments unsuitable for the electrical components, only the flexible sniffer probe can be positioned at the desired position, whereas

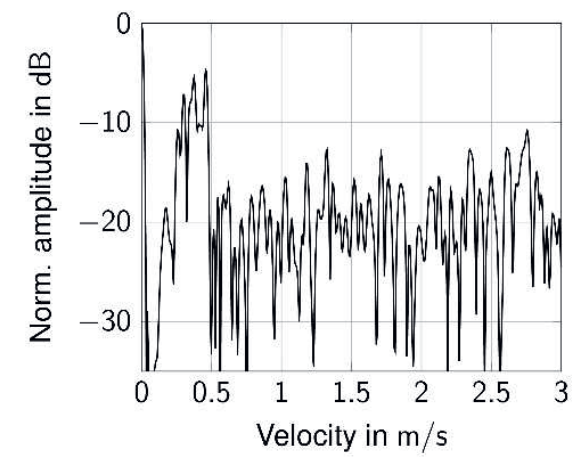

Fig. 4: Volume flow measurement with a sniffer probe in a pipe. Only a small hole for the antenna is needed. The measured water velocity with multiple peaks around $0.4 \mathrm{~m} / \mathrm{s}$ due to turbulent stream current is shown right. 

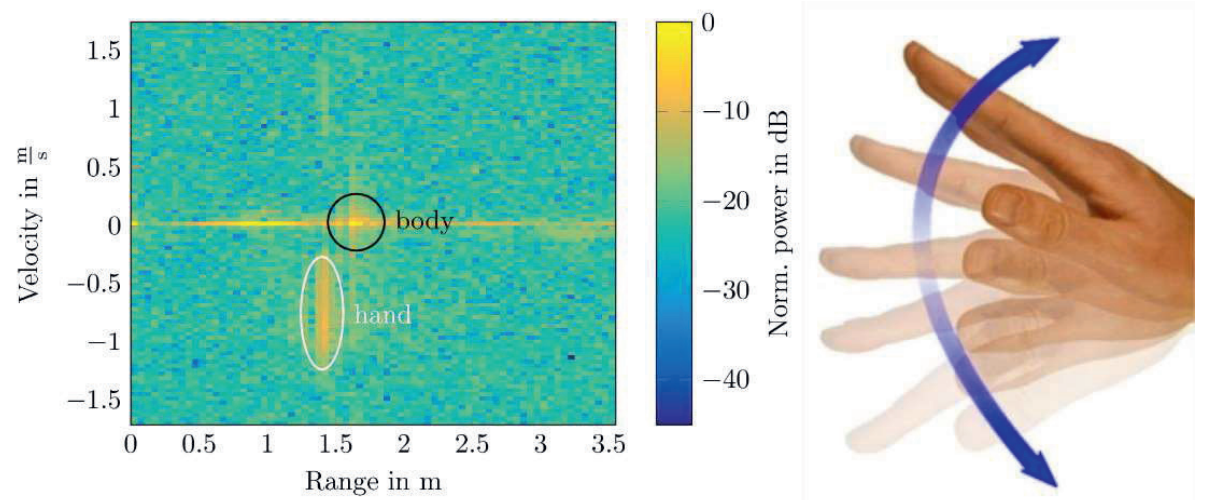

Fig. 5: Range-Doppler diagram for a snapshot during a wipe gesture.

the rest of the radar is placed at a fixed position outside the environment.

Recent research has shown, that with dielectric lenses stacked on flexible dielectric waveguides high gain and mechanical flexible antennas are feasible around $160 \mathrm{GHz}$ [25]. The antenna with a suitable transition from a $160 \mathrm{GHz}$ bistatic radar MMIC to the antenna is shown in Fig. 4 left. Here a volume flow measurement setup in a small pipe with measurement results is depicted. The high operating frequency offers a large bandwidth of $16 \mathrm{GHz}$ and consequently a high spatial resolution of $9.7 \mathrm{~mm}$ [26].

The sniffer probe, antenna and waveguide, is made of high density polyethylene (HDPE) and has low measured losses $(4.5 \mathrm{~dB} / \mathrm{m})$. Compared to a rectangular waveguide with theoretical losses of $8 \mathrm{~dB} / \mathrm{m}$ it is a considerable improvement. For a gain of $24 \mathrm{dBi}$, the antenna has a diameter of only $1 \mathrm{~cm}$.

\section{Human Gesture Recognition with a $77 \mathrm{GHz}$ Radar}

The demand for smart applications in everyday life with touch screens and linked remote control has also increased the interest in human gesture recognition, where camera based systems and computer vision algorithms are popular and yet available. But also a radar system is able to determine the velocity and the position of a target in a room, even more robust and with a higher update rate.

A multi-channel radar system with an increased MIMO 2D-aperture for high angular resolution is able to detect the human hand as a target despite the low radar cross section (RCS) [27]. With an available bandwidth of $3 \mathrm{GHz}$ around $77 \mathrm{GHz}$, a range resolution of $5 \mathrm{~cm}$ is reached. The chirp-sequence modulation enables a velocity resolution up to $10 \mathrm{~cm} / \mathrm{s}$ with an update rate of $20 \mathrm{~Hz}$. Therefore, 512 ramps with a duration of $100 \mu$ s are used. In Fig. 5 a rangeDoppler diagram for a snap shot during a wipe gesture is shown. In addition to the body reflections with $v \approx 0 \mathrm{~m} / \mathrm{s}$ due to the constant position, reflections from the hand are visible. The broad peak in the velocity domain results from many scattering centers at the hand surface and their different velocities.

\section{Altimeter Measurement for UAV at $77 \mathrm{GHz}$}

UAVs have attracted strong interest in the last years. To fly autonomously it is essential to capture the environment and detect obstacles. A radar system can be used as an altimeter.

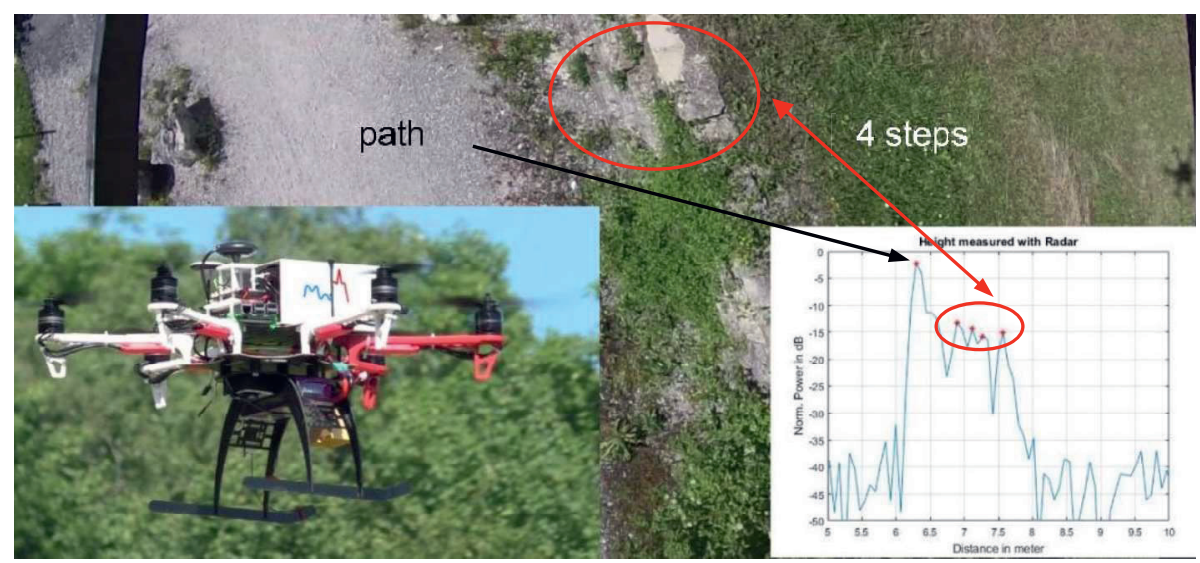

Fig. 6: Radar altimeter at $77 \mathrm{GHz}$ attached to a hexacopter flying over a stepped stone wall. All four steps can be clearly identified in the radar measurement results. 

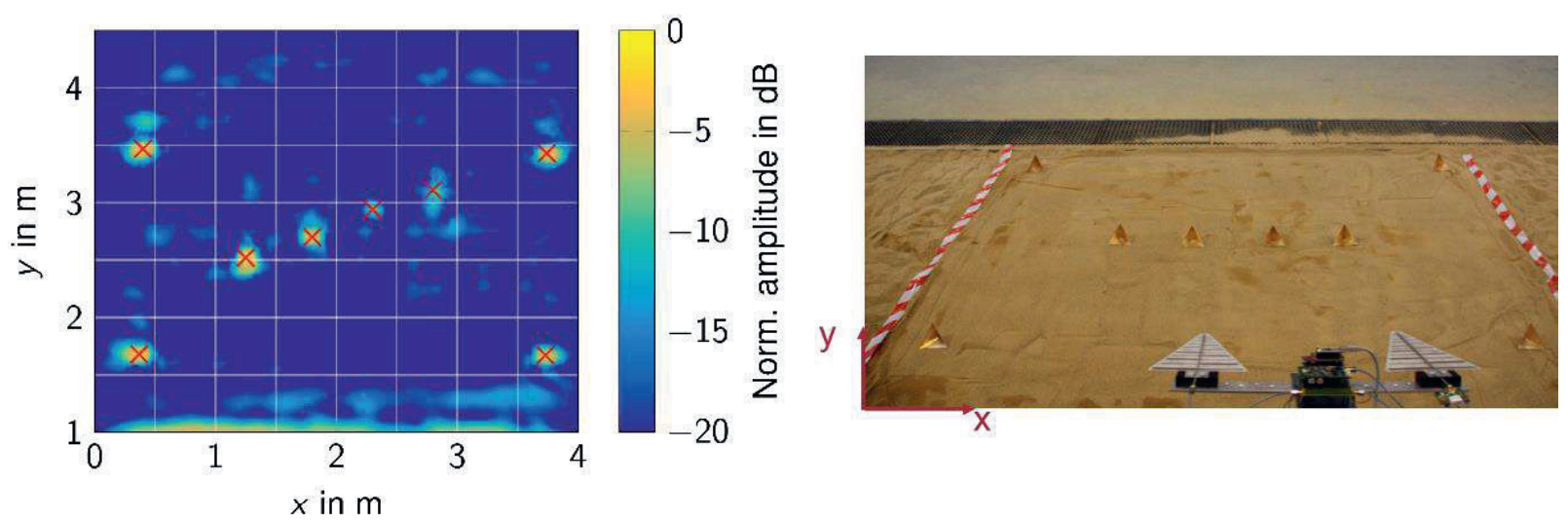

Fig. 7: SAR image (left) for the measured scenario (right) with buried corner reflectors.

Besides the altitude over the current terrain, it is possible to differentiate the terrain between vegetation and building.

Altimeter measurements are performed with a $77 \mathrm{GHz}$ FMCW radar mounted on a UAV. The maximum measured altitude by a constant false alarm rate (CFAR) algorithm is larger than $35 \mathrm{~m}$. The different overflown terrain and vegetation scenarios are visible in the radar response due to different signal-to-noise ratio (SNR) and multiple targets. A tree provides a wide clutter in the radar response whereas multiple targets are visible over a stepped stone wall like shown in Fig. 6. Even between crop and usual grass can be differentiated because of broadened peaks. Compared to other altitude measurement systems, the $77 \mathrm{GHz}$ radar system with a range resolution of $7.5 \mathrm{~cm}$ has obvious advantages. Other possibilities to measure the altitude are GPS systems or pressure altimeter. Only very expensive differential GPS systems reach comparable accuracy. For pressure altimeter rotor blade vorticity leads to inaccuracies. Furthermore, one can just detect the relative altitude to the start point.

\section{Landmine Detection with Ground Penetrating Radar}

Almost every day civilians fall victim to landmines and explosives in former war zones. People try to detect buried landmines with handsets in impassable terrain risking their life.

At frequencies between $1 \mathrm{GHz}$ and $4 \mathrm{GHz}$ microwaves penetrate in the ground up to several centimeters. Therefore, it is possible to detect buried objects like landmines with a radar system and differentiate them from the surrounding ground. With a radar system mounted on a UAV, even impassable terrain like in forests or marshes can be examined on explosives and if necessary demined without risking life. All the flight data (velocity, gear angle, ...) have to be considered in a synthetic aperture radar (SAR) signal processing algorithm to receive a high resolution radar image.

In Fig. 7 a measured SAR image with buried landmine dummies is shown. During the measurement the radar is moved on a straight rail.

\section{Conclusion}

Four new application scenarios for radar sensors have been presented, a $160 \mathrm{GHz}$ flexible sniffer probe, human gesture recognition with a $77 \mathrm{GHz}$ sensor, a $77 \mathrm{GHz}$ radar altimeter mounted to a UAV, and a ground penetrating radar from $1 \mathrm{GHz}$ to $4 \mathrm{GHz}$. This underlines the manifold application areas of radar. The measurement robustness and cheap radar MMICs make radar systems more interesting for industrial applications.

\section{References}

[1] P. Hoogeboorn, H. Hommel, G. Canafoglia, J. Hausle, J. Hubeek, L. Lopez, R. Nachman, and J. Neuville, "TCAR, the Transatlantic Cooperative AGS Radar: a programmatic and technical overview," in First European Radar Conference (EuRAD), Oct. 2004, pp. 145-148.

[2] M. Schikorr, A. Domann, G. Wolf, M. Wranik, and G. Vallant, "TRS-4D Radar: Test Program and Use of Processing Reference Model," in 11th European Radar Conference (EuRAD), Oct. 2014, pp. 313-316.

[3] M. Skolnik, G. Linde, and K. Meads, "Senrad: An Advanced Wideband Air-Surveillance Radar," IEEE Transactions on Aerospace and Electronic Systems, vol. 37, no. 4, pp. 1163-1175, Oct. 2001.

[4] M. Yeary, B. L. Cheong, J. M. Kurdzo, T. y. Yu, and R. Palmer, "A Brief Overview of Weather Radar Technologies and Instrumentation," IEEE Instrumentation Measurement Magazine, vol. 17, no. 5, pp. 10-15, Oct. 2014.

[5] B. Isom, R. Palmer, R. Kelley, J. Meier, D. Bodine, M. Yeary, B. L. Cheong, Y. Zhang, and T. Y. Yu, "The Atmospheric Imaging Radar: System Validation and Observations of Severe 
Weather," in IEEE Radar Conference, May 2012, pp. 0681-0686.

[6] W. Pitz and D. Miller, "The TerraSAR-X Satellite," IEEE Transactions on Geoscience and Remote Sensing, vol. 48, no. 2, pp. 615-622, Feb. 2010.

[7] J. Bock, H. Schafer, K. Aufinger, R. Stengl, S. Boguth, R. Schreiter, M. Rest, H. Knapp, M. Wurzer, W. Perndl, T. Bottner, and T. F. Meister, "SiGe Bipolar Technology for Automotive Radar Applications," in Proceedings of the Bipolar/BiCMOS Circuits and Technology Meeting, Sep. 2004, pp. 84-87.

[8] J. Hasch, E. Topak, R. Schnabel, T. Zwick, R. Weigel, and C. Waldschmidt, "Millimeter-Wave Technology for Automotive Radar Sensors in the $77 \mathrm{GHz}$ Frequency Band," IEEE Transactions on Microwave Theory and Techniques, vol. 60, no. 3, pp. 845-860, Mar. 2012.

[9] M. Furqan, F. Ahmed, R. Feger, K. Aufinger, and A. Stelzer, "A 120-GHz Wideband FMCW Radar Demonstrator Based on a Fully-Integrated SiGe Transceiver with Antenna-in-Package," in IEEE MTT-S International Conference on Microwaves for Intelligent Mobility (ICMIM), May 2016, pp. 14.

[10] A. Fischer, Z. Tong, A. Hamidipour, L. Maurer, and A. Stelzer, "77-GHz Multi-Channel Radar Transceiver With Antenna in Package," IEEE Transactions on Antennas and Propagation, vol. 62, no. 3, pp. 1386-1394, Mar. 2014.

[11] Data Sheet IPM-165, InnoSent GmbH, Am Rödertor 30, 97499 Donnersdorf GERMANY.

[12] H. Meinel and J. Dickmann, "Automotive Radar: From Its Origin to Future Directions," Microwave Journal, vol. 56, no. 9, pp. 24-40, Sep. 2013.

[13] D. Brumbi, "LOW POWER FMCW RADAR SYSTEM FOR LEVEL GAGING," in IEEE MTT-S International Microwave Symposium Digest, vol. 3, Jun. 2000, pp. 1559-1562.

[14] D. Brumbi and I. V. Zyl, "Modern technologies for level measurement," in SENSOR+TEST Conference, 2009.

[15] P. Pahl, T. Kayser, M. Pauli, and T. Zwick, "Evaluation of a High Accuracy Range Detection Algorithm for FMCW/Phase Radar Systems," in European Radar Conference (EuRAD), Sep. 2010, pp. 160-163.

[16] G. Vinci, S. Linz, S. Mann, S. Lindner, F. Barbon, R. Weigel, and A. Koelpin, "A Six-Port Radar System for Precise Distance Measurements and Vibration Monitoring in Industrial Environments," in 17. ITG/GMA Symposium; Proceedings of Sensors and Measuring Systems, Jun. 2014, pp. 1-5.

[17] C. Fischer and W. Wiesbeck, "An Evaluation of Sensor Configurations for Ground Penetration Radar," in IEEE International Geoscience and Remote Sensing Symposium (IGARSS), vol. 3, 2000, pp. 993-995.
[18] J. Laviada, A. Arboleya, F. López-Gayarre, and F. Las-Heras, "Broadband Synthetic Aperture Scanning System for Three-Dimensional Through-the-Wall Inspection," IEEE Geoscience and Remote Sensing Letters, vol. 13, no. 1, pp. 97-101, Jan. 2016.

[19] C. Schroeder and H. Rohling, "X-band FMCW Radar System with Variable Chirp Duration," in IEEE Radar Conference, May 2010, pp. 12551259.

[20] V. Winkler, "Range Doppler detection for automotive FMCW Radars," in European Radar Conference (EuRAD), Oct. 2007, pp. 166-169.

[21] B. Huder, Einführung in die Radartechnik. Stuttgart: Teubner, 1999.

[22] P. Stoica and K. C. Sharman, "Maximum Likelihood Methods for Direction-of-Arrival Estimation," IEEE Transactions on Acoustics, Speech, and Signal Processing, vol. 38, no. 7, pp. 1132-1143, Jul. 1990.

[23] R. Schmidt, "Multiple Emitter Location and Signal Parameter Estimation," IEEE Transactions on Antennas and Propagation, vol. 34, no. 3, pp. 276-280, Mar. 1986.

[24] P. Häcker and B. Yang, "Single snapshot DOA estimation," Advances in Radio Science, vol. 8, pp. 251-256, 2010. [Online]. Available: http://www.adv-radio-sci.net/8/251/2010/

[25] M. Geiger, M. Hitzler, J. Iberle, and C. Waldschmidt, "A Dielectric Lens Antenna Fed by a Flexible Dielectric Waveguide At $160 \mathrm{GHz}$," accepted at: European Conference on Antennas and Propagation (EuCAP), Mar. 2017.

[26] M. Hitzler, S. Saulig, L. Boehm, W. Mayer, W. Winkler, N. Uddin, and C. Waldschmidt, "Ultracompact 160-GHz FMCW Radar MMIC With Fully Integrated Offset Synthesizer," IEEE Transactions on Microwave Theory and Techniques, vol. PP, no. 99, pp. 1-10, 2017.

[27] P. Hügler, M. Geiger, and C. Waldschmidt, "RCS Measurements of a Human Hand for RadarBased Gesture Recognition at E-band," in German Microwave Conference (GeMiC), Mar. 2016, pp. 259-262. 